\title{
Conus Terminallis Neurocysticercosis: A Rare Cause of Lumbar Radiculopathy
}

Andreia SSBT Veiga ${ }^{1 *}$, Andreia FMR Matas ${ }^{1}$, Joao PMS Gabriel ${ }^{1}$ and Mário Resende Martins ${ }^{2}$

${ }^{1}$ Neurology Department, Centro Hospitalar de Trás os Montes e Alto Douro, Vila Real, Portugal

${ }^{2}$ Neurosurgery department, Centro Hospitalar de Vila Nova de Gaia-Espinho, Portugal

*Corresponding author: Andreia Veiga, Centro Hospitalar de Tras os Montes e Alto Douro, Vila Real, Portugal, Fax: 00351259300514; E-mail: andreia.a.veiga@gmail.com

Received date: Oct 30, 2014, Accepted date: Jan 08, 2015, Published date: Jan 15, 2015

Copyright: (C) 2015 Veiga A, et al. This is an open-access article distributed under the terms of the Creative Commons Attribution License, which permits unrestricted use, distribution, and reproduction in any medium, provided the original author and source are credited.

\begin{abstract}
Introduction: Neurocysticercosis (NCC) is a parasitic infection caused by the larvae of Taenia solium. Spinal neurocysticercosis presentation is an uncommon clinical condition especially if the only segment involved is cauda
\end{abstract} equine.

Case report: We present a 57 year old woman with a history of a lumbar radiculopathy in which the lumbar MRI showed an expansive lesion at conus terminallis level. The lesion was excised revealing parasitic cisticerci. An albendazol cycle was performed and 1 year after treatment she remains asymptomatic and no more lesions were identified.

Discussion: NCC is a rare condition, especially at the sacral region, and the diagnosis is difficult due to the nonspecificity of symptoms being this case so relevant to resemble this entity in non-endemic areas like ours.

Keywords: Conus terminallis; Parasitic cysticerci; Radiculopathy

\section{Introduction}

Neurocysticercosis (NCC) is a parasitic infection caused by the larvae of Taenia solium which affects humans mainly by accidental ingestion of eggs containing infective oncosferes. Its frequency in non endemic areas is increasing justified by migratory fluxes. Spinal neurocysticercosis presentation is an uncommon clinical condition, especially if the only segment involved is the cauda equina and can lead to irreversible neurological deficits if untreated. We present a case of neurocysticercosis in which the only symptom was a lumbar radiculopathy.

\section{Case Report}

57 year old woman with a history of low back pain evolving for 4 months. She denied urinary and gastrointestinal disturbance, sensory or motor symptoms. She also had no fever or other systemic features. No relevant medical past to highlight. At neurological exam no relevant abnormalities were found including absence of sensory or motor deficits; only a positive left Lasegue sign and a left aquilian reflex diminished, suggesting a possible lumbar radiculopathy. Lower limbs electromyography confirmed a left L5-S1 radiculopathy, showing chronic neurogenic changes in the muscles supplied by these roots (motor unit action potential with a poliphasic pattern, high amplitude and poor recruitment in anterior tibialis, peroneus longus and gastrocnemius muscles; no spontaneous motor activity was found). Lumbar MRI showed an expansive lesion at the caudal end of lumbar canal, iso-intense to CSF in T1 sequences, with an heterogeneous signal intensity in $\mathrm{T} 2$ sequences and homogeneous gadolinium enhancement, suggesting ependymoma (Figure 1). Two weeks after the diagnosis a L4-S1 laminectomy was performed to excise the lesion that macroscopically was totally resected. Anatomopathological examination showed parasitic cysticerci with inflammatory infiltrate and necrotic areas (Figure 2). She was treated with albendazol $800 \mathrm{mg} /$ day during 30 days. Brain MRI revealed multiple calcifications suggestive of an old process of neurocysticercosis and cervico-dorsal MRI was unremarkable. CSF study performed 5 months after surgery was normal, including negative serology for $T$. solium. One year after surgery she remains asymptomatic and a lumbar MRI performed at that time showed no recrudescent lesions. Non endemic history was found.

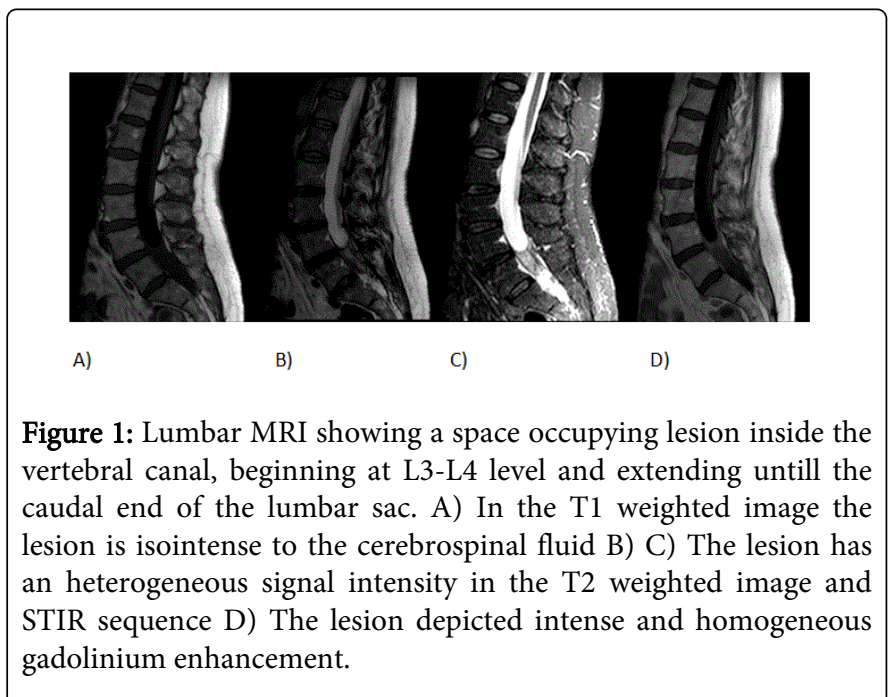


Citation: Veiga A, Matas A, Gabriel J, Martins MR (2015) Conus Terminallis Neurocysticercosis: A Rare Cause of Lumbar Radiculopathy. J Neurol Neurophysiol 6: 265. doi:10.4172/2155-9562.1000265

Page 2 of 2

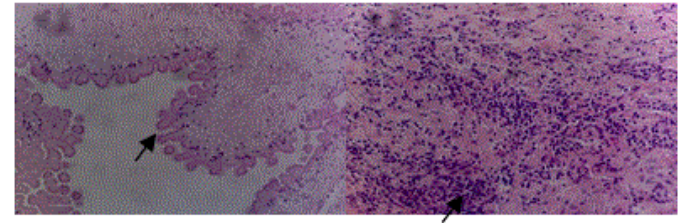

A)

C)

Figure 2: Anatomopathological examination of the lesion (hematoxylin eosin staining) A)x 40; cross section of lesion showing parasitic cisticerci (arrow in the membrane of cyst); C)x100; inflammatory cells (arrow)

\section{Discussion}

Although NCC is the most frequent parasitic infection of the central nervous system, tipically affecting brain parenquima, subarachnoid spaces and ventricular system, the spinal involvement is rare $[1,2]$ even in endemic areas, with an estimated prevalence of 0,7 to $5,85 \%$ [3]. The cysticerci spreads through arterial blood circulation which explain why in spinal segments the thoracic region is the most frequently affected. These cause inflammatory reaction and mass effect responsible for the symptoms [3]. The most common clinical manifestations are secondary to medullar compression (paraparesis, urinary and bowel dysfunction), being this case an exceptional presentation as an isolated lumbar radiculopathy result of a single lesion in an extreme unusual place as conus terminallis. The majority of reported cases of NCC at medulary level ranged between 20-45 years of age, and symptoms duration varied from one week to 10 years [3]. The diagnosis is suspected by MRI but the lesion has no specific features and the differential diagnosis includes neoplastic, inflammatory, demyelinating, vascular and granulomatous lesions so surgery is necessary to define the etiology. Like in our case surgical resection was mandatory because a tumor was suspected in lumbar MRI. Although primarily surgical, the treatment should be complemented with a regimen of medication because of the difficulty to remove the entire lesion. In our case one year after treatment CSF and lumbar MRI were negative so we consider the disease controlled

In sum, NCC is a rare condition, especially at the sacral region, and the diagnosis is difficult due to the non-specificity of symptoms being this case so relevant to resemble this entity in non-endemic areas like ours.

\section{References}

1. Agale SV, Bhavsar S, Choudhury B, Manohar V (2012) Isolated intramedullary spinal cord cysticercosis. Asian J Neurosurg 7: 90-92.

2. Agrawal R, Chauhan SP, Misra V (2012) Focal spinal intramedullary cysticercosis. Acta Biomed. 2008 Apr; 79(1):39-41. West J Emerg Med 13: 434-436.

3. Guedes Corrêa J, Macedo R, Vaitsman R, Mattos J, Agra J (2006) Intramedullary spinal cysticercosis simulating a conus medullary tumor. Arq Neuropsiquiatr 64: 149-152. 